\title{
Haltungsbegriffe und Begriffshaltungen
}

Da forderst Du von mir eine Lektion in angewandter philosophischer Haltungskunde, eine Lektion in geistigem Buckelturnen. Zunächst einmal ist Haltung ein sehr deutscher Begriff mit sehr deutschem Pathos. Ins Schweizerdeutsche lässt er sich zur Not noch übersetzen - da sind wir Schweizer erstaunlich deutsch -, aber schon mit dem Englischen und dem Französischen wird es schwierig. Dort meint «attitude» zwar

48 Haltung im Sinne von Einstellung, aber es fehlt der "attitude», egal, ob englisch oder französisch, nicht nur das Rückgrat der Körperhaltung, sondern auch die heroische Konnotation des Standhaltens. Und dann hätten wir die «hexis» der alten Griechen, die über die Römer als «habitus» auf uns kam. Gemeint ist das, was jemand hat, "die Habe», um ein schönes altes Wort zu rehabilitieren. Also etwas, das zu einem gehört, das man erworben hat. Und diese «hexis» spielt in der Ethik seit jeher eine Rolle. So bestimmt beispielsweise Aristoteles die Tugend als «hexis» der guten Mitte zwischen den Extremen. «Hexis» ist nichts, was einfach so gegeben wäre. Man erwirbt diese Habe im Laufe des Lebens und sie muss keineswegs etwas Positives sein. Auch das Laster ist eine «hexis», ein andauerndes Verhalten, eine Gewohnheit.

Eine Haltung muß man sich erwerben, sie akkumuliert mit den Erfahrungen, die man im Lauf der Jahre macht. Vorausgesetzt man will daraus mehr als nur pragmatische Schlüsse ziehen. Ein Bedürfnis nach Haltung zu entwickeln, die situationsunabhängig gilt, a priori, ist etwas anderes. Aber können wir nicht zu Deiner Haltung als Philosoph kommen?

Ein bisschen mehr Anlauf brauche ich dazu freilich. Zumal die Haltung als starker philosophischer Begriff ohnehin erst ein Kind der letzten Zwischenkriegszeit ist. Und der Kontext, in dem er stark gemacht wird, ist nicht unbedingt appetitlich. Prominent hat ihn Erich Rothacker, Habermas' Doktorvater, herausgestellt, in seiner 1934 publizierten «Geschichtsphilosophie», die nicht nur den «grossen Staatsmann Adolf Hitler» feierte und den «Rassegedanken» als unabdingbar ausgab. Sondern auch intensiv über Haltung nachdachte, nämlich als «Dauerantwort auf eine Dauerlage»: «Haltungen sind menschliche 
Seinsweisen, in denen der Mensch sich wollend und vorsätzlich hält und deren Innehaltung und Höherentfaltung mit seiner Ehre, seinem Stolz, seiner Selbsteinschätzung, seinen Ansprüchen an Würde, Rang und Adel unlösbar verknüpft ist.» Haltung um der Haltung willen. Der Existenzphilosoph Karl Jaspers hat versucht, Paroli zu bieten, und veranschaulicht, wie in jeder Haltung die Gefahr lauert, die für Menschen charakteristische Offenheit zu vernichten. Die Gefahr, die Du «Erstarren» nennst. Jaspers will nur «provisorische Daseins-Haltungen» gelten lassen, die der Existenz keine letzte, feste Form geben: «Haltung, verabsolutiert, macht starr und tot.»

Mit Jaspers rennst Du bei mir offene Türen ein. Aber wolltest Du nicht über Deine eigene Haltung reden? Vorhin hast Du Dich, vorsichtig zwar, als Konservativer zu erkennen gegeben; häufig jedoch auch schon als Sophist, der Haltungen nur vorübergehend, vielleicht aus rhetorischem Ehrgeiz, vertritt, statt sie tatsächlich einzunehmen. Wie geht das zusammen?

\section{Gewohnheit versus Haltung}

Nicht verweigern, nicht verzichten, nur bereit sein, seine Haltungen immer wieder zur Disposition zu stellen. Vielleicht könnten wir, wenn wir an einem positiven Begriff von Haltung festhalten wollen, sie von Gewohnheit unterscheiden. Gewohnheiten sind oft nützlich, weil sie unser Leben vereinfachen. Sie helfen uns, nicht in jeder Lebenssituation immer wieder neu darüber nachdenken zu müssen, was wir jetzt machen sollen. Aber Gewohnheiten verstellen oft genug den Blick, wo es nötig wäre, tatsächlich einmal nachzudenken. Häufig genug sind Gewohnheiten passiv, antrainiert, einem zugewachsen, ohne dass wir selbst ihr Wachsen und Wuchern unter Kontrolle hätten. Gegen diese blosse Gewohnheit könnten wir die Haltung in Stellung bringen, als etwas Aktives, von uns Gewähltes. Man nimmt Haltungen ein, gewinnt sie. Als das, was unserem Leben Form gibt. Als das, womit wir unserem Leben Form geben. Und ihr gewaltiger Vorteil ist es, dass wir 